\title{
AUTOMATIC STEERING FOR THE CTF3 LINEAR ACCELERATOR*
}

\author{
Ronen Lifshitz, Technion - Israel Institute of Technology, Haifa, Israel \\ Daniel Schulte, CERN, Geneva, Switzerland
}

\begin{abstract}
A system for automatic beam steering has been developed and installed in the CLIC Test Facility - CTF3 linear accelerator at CERN. Using a simple beam position-reading minimisation for trajectory correction, this system has been tested in the 2004 summer run of CTF3. It has already been proven useful as a tool in machine commissioning and operation. In this paper, the CTF3 automatic steering system is introduced, trajectory correction results are presented, and the agreement with a simulation model of the machine is discussed.
\end{abstract}

\section{INTRODUCTION}

The accelerating power in CLIC will be produced by using decelerators to extract power from a low-energy high-current drive beam. The latter will be accelerated in a low-frequency fully loaded linac, allowing close to $100 \%$ efficiency in transforming RF into beam power [1].

The CLIC test facility at CERN (CTF3) is aimed at demonstrating this concept. It will consist of all the main components of the drive beam complex including the drive beam accelerator [2]. This accelerator is based on $3 \mathrm{GHz}$ accelerating structures integrated in a triplet lattice [3] and accelerates a beam of about 3.5A and a pulse length of up to more than a microsecond. With all structures installed, it will accelerate the beam to an energy of $150 \mathrm{MeV}$.

An automatic beam steering system has been installed for the CTF3 linac during the 2004 machine run. The purposes of this system are to supply a useful tool for machine operation and to serve as a benchmark for a simulation model, which is written using the tracking code PLACET [4]. Beam trajectory correction can be performed either by using a measured response of the trajectory to corrector dipoles or a simulated one, as is detailed below.

\section{THE SOFTWARE}

The CTF3 linac has been modelled using the PLACET code and also the correction techniques have been implemented using this tool. PLACET is an extension of the TCL [5] language and can be controlled completely via TCL-scripts. Consequently the response matrix measurement and correction methods have been implemented in TCL. The access to hardware has been realised using java, as explained below.

\section{Correction Technique}

The correction method aims at minimising the RMS

\footnotetext{
*Work supported by the European Community Research Infrastructure Activity under the FP6 "Structuring the European Research Area" programme (CARE, contract number RII3-CT-2003-506395).
}

beam offsets in the BPMs along the accelerator using corrector dipoles to steer the beam. One can, with good precision, assume that the beam position changes linearly with the current in these correctors. Hence one can construct a response matrix $\mathrm{R}$ that allows calculation of changes in BPM readings as a function of the correctors. A number of correction methods are implemented in PLACET; for CTF3 the simplest one has been used. Based on the BPM readings and the response matrix, it determines the combination of corrector settings which minimise the RMS orbit offset. The corrector strengths are then changed accordingly. Usually only a fraction of the changes is applied (typically 50\%); this makes the system less sensitive to errors in the response matrix.

The software allows using subsets of devices (dipoles, BPMs, or both) for trajectory correction, so that optimising the machine by parts is possible using one matrix.

\section{Interface to Hardware}

The program package needs to access the hardware in order to read the BPMs and set the corrector strengths; this is required for the measurement of the response matrix as well as for the correction procedure itself.

A dedicated hardware-access console was implemented in JAVA, using the ASC [6] toolbox, which is the standard tool for accessing machine control equipment in the PS complex at CERN. A corresponding toolbox was written in TCL, which communicates with the JAVAbased console and allows an indirect control over machine equipment from within TCL.

As a result of past experience, an automatic logging of various machine parameters is now a part of the steering software. This allows feeding measured property values into the simulation as inputs. Examples for such properties are the measured beam current and RF power.

\section{TRAJECTORY CORRECTION RESULTS}

The steering software was installed in CTF3 during the 2004 CTF3 runs. During these runs the beam has been sent through the linac as well as into a special beam line, the so-called high power test stand. In this beam line a special power extraction and transfer structure (PETS) has been installed to extract significant RF power from the drive beam. For both beamlines the code package was used to first measure the response matrix and then to correct the beam trajectory.

In order to determine the response matrix, the current supply to each one of the corrector magnets is scanned through a range of values, and the BPM readings are logged during this process. Figure 1 shows the data logged for one, while the dipole corrector upstream of it was scanned. The response to the corrector is quite linear. 
From this data the response matrix has been constructed using linear fits.

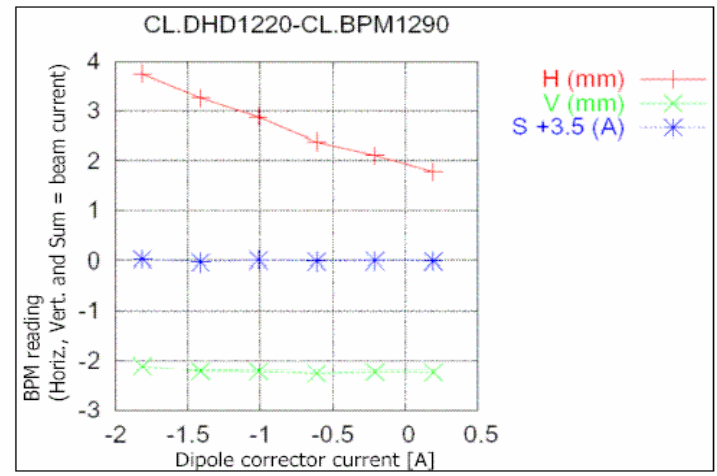

Figure 1: BPM signals while varying a corrector magnet current. DHD1220 is a horizontally deflecting dipole. BPM 1290 is about 3 meter downstream of it.

Figure 1 shows an example of a successful trajectory optimisation in the CTF3 PETS line using a previously determined response matrix. Four horizontal BPM readings are shown, during five iterations of the correction procedure. The signal convergence into the beam axis is clearly seen.

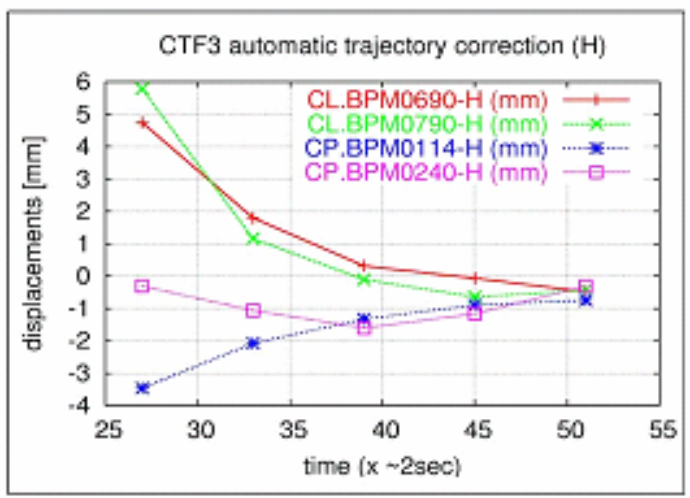

Figure 2: Horizontal trajectory correction in the CTF3 PETS line - BPM readings versus time. Four BPM readings are shown. The beam converges from up to six to less than one millimetre off-axis for all BPMs.

\section{SIMULATION AND MEASUREMENT}

While the correction based on the measured response matrix worked very well, It remains to be determined whether one can derive the response matrix by simulation. To this end a detailed model of the CTF3 linac has been set up using the simulation code PLACET. The response matrix measurement procedure has been implemented within the code, so that a comparison of the simulated and the measured results is possible.

\section{Beam Energy Estimation}

The beam energy is estimated in a simple way by kicking the beam in a dipole corrector and measuring the beam offset in the next BPM. Having no quadrupole between them, this estimation is independent of any uncertainty related to the focusing in the linac.

Calibration of the CTF3 corrector magnets has been done, and the resulting deflection $\alpha$ satisfies [7]:

$$
\alpha p=(65.4 I \pm 19.6) \frac{\mathrm{mrad} \cdot \mathrm{MeV}}{\mathrm{C}}
$$

Here, $p$ is the beam momentum and $I$ is the current. In the following the error term has been ignored, since it is dominated by the hysteresis effect [7].

The results of the beam energy measurements and simulations are shown in Figure 3. The simulation starting point is approximately at the CTF3 injector position [2].

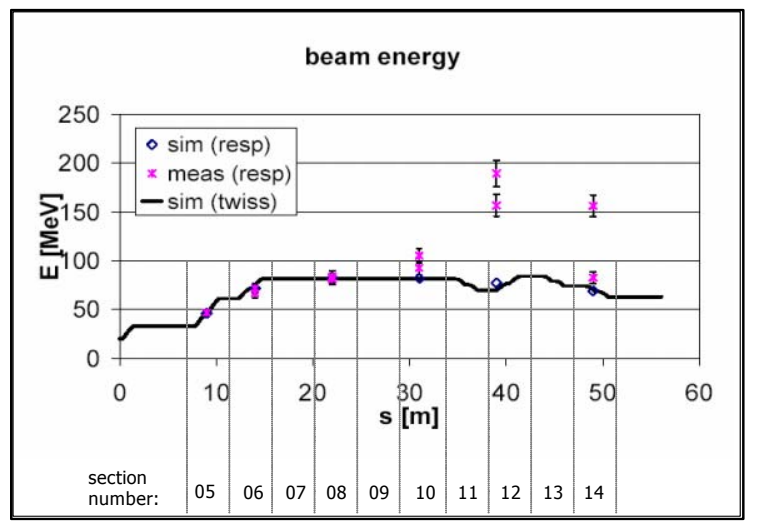

Figure 3: Beam energy estimation (no focusing). A continuous energy profile is provided by the simulation. Discrete points represent the measurement results, and simulation results which correcpond to the procedure used in the measurement. Measured values come in pairs due to the horizontal and vertical responses used for each corrector-BPM pair.

The simulation provides both a continuous energy profile and discrete points, which correspond to the simulation of the exact procedure used for measurement. The power in the different accelerating structures used in the simulation is shown in Table 1.

Table 1: RF power readings, as measured during response measurement in CTF3 and used in simulations

\begin{tabular}{|l|c|c|c|c|}
\hline Section: & 03 & 05 & 06 & \\
\hline $\begin{array}{l}\text { Power: } \\
\text { (MW/structure) }\end{array}$ & 65.55 & 63.81 & 41.32 & \\
\hline & & & & \\
\hline Section: & 11 & 12 & 13 & 14 \\
\hline $\begin{array}{l}\text { Power: } \\
\text { (MW/structure) }\end{array}$ & 0.008 & 27.06 & 0.12 & 0.01 \\
\hline
\end{tabular}

Figure 3 shows a good agreement between simulation and measurement in the first part of the machine, while differences appear after section 09. The small difference at section 10 could be explained by various modelling or instrumentation errors. However, for the next two BPMs very large differences of about a factor two between simulation and measurement are observed. It is not obvious how this large discrepancy can be explained by 
an error in beam energy modelling. During preliminary runs in 2003, a BPM failure has been detected by an error in energy estimation using the method described above. However, the reason for the current disagreement is yet unknown and under study.

\section{Response Coefficients Comparison}

Following the evident agreement in energy estimation along the first part of the linac, a comparison of response coefficients has been done along the same linac segment. For this comparison the responses of each BPM to each corrector are taken into account, so that focusing effects are included. Figure 4 shows the measured response coefficients versus the simulated ones. The correlation between these values seems quite reasonable, suggesting that the agreement between model and reality is not too bad for this part of the linac.

The original results of this comparison suggest a scaling correction to the simulation. After the application of an overall scaling of 1.125 to the lattice focusing strength, the results improve significantly (see Figure 4). This effect is also evident in the reconstruction of betatron phase advance, as described below.

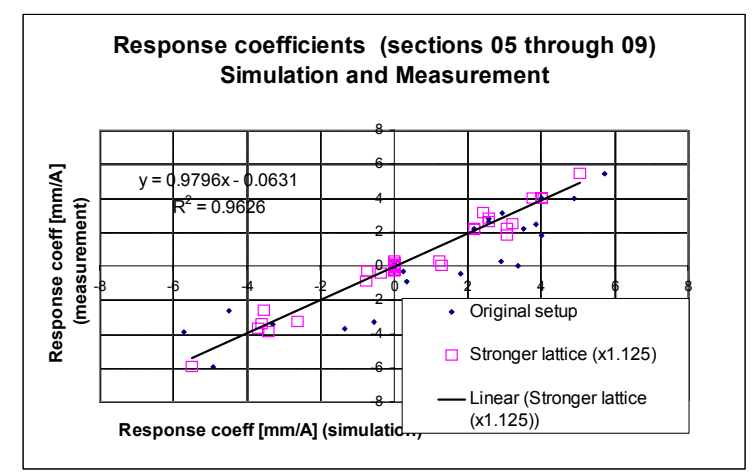

Figure 4: Response coefficient comparison: measured values are plotted against simulated ones. Notice the high correlation and the good agreement to a straight line with a unit slope. Results improved after the application of an overall scaling factor to the lattice focusing.

\section{Correlation in the Transverse Position}

Following significant jitter observed in BPM readings during measurement, a BPM to BPM correlation analysis has been performed. It showed high correlations between measured signals in many cases, approaching full correlation in some. This suggests that the jitter source is the beam itself. Consequently an analysis of the corresponding trajectories has been performed.

As can be seen in Figure 5 the different trajectories in the horizontal plane are well predicted by simulation. The analysis suggests that a transverse beam jitter is introduced along the injector. In addition, there seems to be an additional jitter source inside the linac, in the approximate location of the CTF3 chicane magnets [2]. In the vertical plane the beam jitter is much smaller.

While optimising the agreement in betatron wavelength along the linac, an overall scaling of 1.125 has been applied to the lattice focusing strength. This change was favoured by the agreement in response coefficient in the first part of the linac (see above). The good agreement seen in Figure 5 indicates that the model reproduces the phase advance along the linac quite well and probably only small corrections are required.

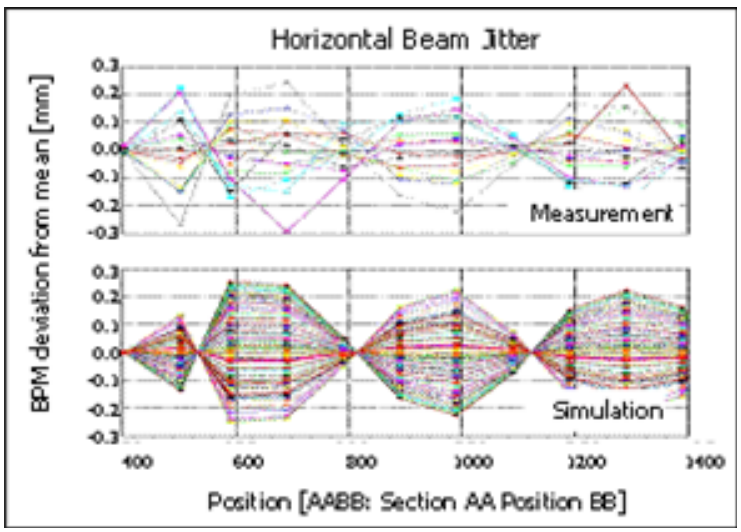

Figure 5: Beam jitter in CTF3 - simulation and measurement.

\section{CONCLUSION}

An automatic steering response measurement system has been implemented in the CTF3 LINAC at CERN. This system has been proven useful as a trajectory optimisation tool. The measurement results are used for comparison to the machine simulation model, in order to benchmark the model itself and to improve the performance by replacing the measurement with a calculation. The agreement between the model and the measurements was shown to be reasonable along a limited portion of the linac. The extention of this agreement further downstream the machine is an ongoing study.

\section{REFERENCES}

[1] The CLIC STUDY of a MULTI-TeV e +e -LINEAR COLLIDER / J.P. Delahaye; Proceedings of the 1999 Particle-Accelerator Conference, N.Y.

[2] CTF3 Design Report / G. Geschonke \& A. Chigo; CERN/PS 2002-008

[3] Beam Dynamics Simulation for the CTF3 Drive Beam Accelerator / D. Schulte; CERN-PS 2000-049

[4] PLACET - A program to simulate drive beams / D. Schulte; Proceedings of EPAC 2000, Vienna

[5] Tool Command Language - TCL / J. Ousterhout; publicly available at http://www.tcl.tk/

[6] ASC - Applications Services and Components, a JAVA package for controlling accelerators in the PS complex; provided by CERN AB/PS-CO-AP

[7] Measurement and Calibration of the Corrector Magnets and the Chicane Dipole Magnets for CTF3 / O. Forstner; CERN-AB-2003-(ABP) 077

[8] The Automatic Steering System in CTF3 / R. Lifshitz, D. Schulte; CARE/ELAN Document-2005 